\title{
The most important challenges ahead of microbiome pattern in the post era of the COVID-19 pandemic
}

\author{
Hanieh-Sadat Ejtahed ${ }^{1,2} \cdot$ Shirin Hasani-Ranjbar ${ }^{2} \cdot$ Seyed Davar Siadat ${ }^{3,1} \cdot$ Bagher Larijani $^{1}$
}

Received: 18 June 2020 / Revised: 18 June 2020 / Accepted: 26 June 2020 / Published online: 3 July 2020

(C) Springer Nature Switzerland AG 2020

\begin{abstract}
As the ongoing COVID-19 pandemic posed a huge challenge to global public health, emphasis on personal hygiene caused extraordinary daily use of detergents and household cleaning products. Since antibacterial cleaning products have the capacity to change the environmental microbiome, increased exposure to household disinfectants could be associated with alterations in human microbiota composition and function. Moreover, the emerging links between household cleaning products and occurrence of non-communicable diseases and antibiotic resistance may involve the gut microbiome. The mediating effects of disturbed gut microbiota on associations between home use of cleaning products and life-long health consequences have been documented in some observational studies. Regarding the evidence associated reduction in exposure to non-pathogenic commensal bacteria and gut dysbiosis, further study is required to assess the effect of massive use of cleaning products during COVID-19 pandemic on long-term host health mediated by the altered microbiota. In this context, recommendations to consume probiotics and fermented foods might reverse the consequences by alleviating dysbiosis.
\end{abstract}

Keywords COVID-19 $\cdot$ Microbiota $\cdot$ Gut dysbiosis $\cdot$ Disinfectants

Nowadays the world has faced the challenge of a novel coronavirus outbreak. Severe acute respiratory syndrome coronavirus 2 (SARS-CoV-2) with high infectivity has rapidly spread all over the world and it has officially been declared a pandemic by the World Health Organization (WHO) [1]. Governments and the medical community try to take actions to prevent COVID-19 transmission [2]. Besides to public health measures like social distancing and quarantine, adherence to the principles of personal hygiene is a practical recommendation to prevent disease [3]. In this context,

Seyed Davar Siadat

d.siadat@gmail.com

$\triangle$ Bagher Larijani

larijanib@tums.ac.ir

1 Endocrinology and Metabolism Research Center, Endocrinology and Metabolism Clinical Sciences Institute, Tehran University of Medical Sciences, Tehran, Iran

2 Obesity and Eating Habits Research Center, Endocrinology and Metabolism Clinical Sciences Institute, Tehran University of Medical Sciences, Tehran, Iran

3 Department of Mycobacteriology and Pulmonary Research, Microbiology Research Center, Pasteur Institute of Iran, Tehran, Iran compliance with health recommendations like washing hands frequently may have other consequences for our health and well-being. A more alarming issue in coronavirus outbreak is the emphasis on cleanliness and consequent extraordinary daily use of detergents, antiseptics and other cleaning products in the home which could cause dramatic decrease in microbial load on household surfaces. The notable issue in this regard is that frequent use of domestic detergents has the capacity to alter the environmental microbiome [4]. Indeed, cleaning products could not discriminate between useful and harmful bacteria. Moreover, studies have been shown that minimal inhibitory concentrations (MICs) of anti-microbial agents can induce horizontal transfer of antibiotic resistance genes between our resident bacteria [5]. According to the 'Hygiene Hypothesis' although sanitization, urban living and access to antibiotics keep us safe from infectious disease, reduction in exposure to non-pathogenic commensal strains could play a substantial role in the increasing prevalence of allergic diseases [6].

Previous studies presented that low-dose exposure to chemicals in detergents have significant effects on microbiota composition and function [7]. In a preclinical study, microbiome $\alpha$-diversity and fecal concentration of microbiota-derived metabolites including branched chain fatty 
acids and short chain fatty acids decreased dramatically in healthy subjects with dishwashing detergent [8]. It is noteworthy that reduced intestinal microbial diversity has been associated with non-communicable diseases [8,9].

As an underlying mechanism it should be noted that cleaning products contain some chemicals that have been linked to gut dysbiosis. Triclosan, 5-chloro-2-(2,4dichlorophenoxy)phenol, as one of these chemicals is an antibacterial compound in soaps, detergents and hand sanitizers which can be absorbed through the skin $[4,7]$. In these coronavirus pandemic days, exposure to triclosan has been increasing exponentially with raising in detergent use. Triclosan exposure has a profound impact on gut microbiome by inducing perturbations at both compositional and functional levels $[7,10,11]$. Considering that healthy gut microbiome plays an essential role in the body's immune response to infection and in maintaining overall health [12], triclosan exposure may be linked to the adverse effects including endocrine disorders, antibiotic resistance, colonic inflammation and tumorigenesis $[4,13]$. Besides triclosan exposure during early life may also cause disturbances in metabolism and gut microbiota which last a lifetime and accumulated over time [14]. Previous studies indicated the links between household cleaning products and childhood overweight. In the Canadian Healthy Infant Longitudinal Development (CHILD) birth cohort, it has been shown that more frequent home use of disinfectants was associated with increase in abundance of Lachnospiraceae in infant gut microbiota. Relative abundance of Lachnospiraceae was the mediating factor for childhood overweight and obesity [15].

There is currently limited knowledge on the effects of detergents on the human microbiota, and research to date has predominantly occurred in animal models, so further study is required to assess the effect of massive use of cleaning products during COVID-19 pandemic on long-term host health mediated by the altered microbiota. As the ongoing COVID19 outbreak posed a huge challenge to global public health, consuming probiotics and fermented foods might reverse the consequences by alleviating dysbiosis. Probiotics could help in preserving biodiversity of microbiota and attenuating toxic effects of chronic detergent exposure [16]. Although the effect of massive use of cleaning products and its environmental accumulation on our microbiome health is not yet known completely, recommendations to regular use of probiotic and prebiotic products might have possible implications for preventing of gut microbial perturbation.

In conclusion, antiseptic and disinfectant products are expected to be detrimental on microbiome patterns in human, animal and environment. The most understandable of these changes is perturbation of skin and lung microbiota. Thus, the effect of this dysbiosis on COVID-19 complications besides to long-term health should be investigated.
Authors' contributions HSE designed and drafted the manuscript. SHR helped to draft the manuscript. DS and BL edited the manuscript. All authors read and approved the final manuscript.

Availability of data and material Not applicable.

\section{Compliance with ethical standards}

Conflict of interest The authors declare that they have no competing interests.

Ethics approval Not applicable.

Consent to participate Not applicable.

Consent for publication Not applicable.

Code availability Not applicable.

\section{References}

1. Hui DS, Azhar I, Madani E, Ntoumi TA, Kock F, Dar R, et al. The continuing 2019-nCoV epidemic threat of novel coronaviruses to global health - the latest 2019 novel coronavirus outbreak in Wuhan, China. Int J Infect Dis. 2020;91:264-6.

2. Phelan AL, Katz R, Gostin LO. The novel coronavirus originating in Wuhan, China: challenges for global health governance. JAMA. 2020. https://doi.org/10.1001/jama.2020.1097.

3. Xiao Y, Torok ME. Taking the right measures to control COVID19. Lancet Infect Dis. 2020.

4. Sanidad KZ, Xiao H, Zhang G. Triclosan, a common antimicrobial ingredient, on gut microbiota and gut health. Gut Microbes. 2019;10(3):434-7.

5. Jutkina J, Marathe NP, Flach CF, Larsson DGJ. Antibiotics and common antibacterial biocides stimulate horizontal transfer of resistance at low concentrations. Sci Total Environ. 2018;616-617: $172-8$.

6. Bloomfield SF, Stanwell-Smith R, Crevel RW, Pickup J. Too clean, or not too clean: the Hygiene Hypothesis and home hygiene. Clin Exp Allergy. 2006;36(4):402-25.

7. Yee AL, Gilbert JA. Is triclosan harming your microbiome? Science. 2016;353(6297):348-9.

8. Gerasimidis K, Bryden K, Chen X, Papachristou E, Verney A, Roig $\mathrm{M}$, et al. The impact of food additives, artificial sweeteners and domestic hygiene products on the human gut microbiome and its fibre fermentation capacity. Eur J Nutr. 2019. https://doi.org/10. 1007/s00394-019-02161-8.

9. Lambeth SM, Carson T, Lowe J, Ramaraj T, Leff JW, Luo L, et al. Composition, diversity and abundance of gut microbiome in prediabetes and type 2 diabetes. J Diabetes Obes. 2015;2(3):1-7.

10. Gao B, Tu P, Bian X, Chi L, Ru H, Lu K. Profound perturbation induced by triclosan exposure in mouse gut microbiome: a less resilient microbial community with elevated antibiotic and metal resistomes. BMC Pharmacol Toxicol. 2017;18(1):46.

11. Wang C, Yu Z, Shi X, Tang X, Wang Y, Wang X, et al. Triclosan enhances the clearing of pathogenic intracellular salmonella or Candida albicans but disturbs the intestinal microbiota through mTOR-independent autophagy. Front Cell Infect Microbiol. 2018;8:49.

12. Lazar V, Ditu LM, Pircalabioru GG, Gheorghe I, Curutiu C, Holban $\mathrm{AM}$, et al. Aspects of gut microbiota and immune system 
interactions in infectious diseases, immunopathology, and cancer. Front Immunol. 2018;9:1830.

13. Yueh MF, Tukey RH, Triclosan. A widespread environmental toxicant with many biological effects. Annu Rev Pharmacol Toxicol. 2016;56:251-72.

14. Ma Y, Guo Y, Ye H, Zhang J, Ke Y, et al. Perinatal Triclosan exposure in the rat induces long-term disturbances in metabolism and gut microbiota in adulthood and old age. Environ Res. 2020;182:109004.

15. Tun MH, Tun HM, Mahoney JJ, Konya TB, Guttman DS, Becker $\mathrm{AB}$, et al. Postnatal exposure to household disinfectants, infant gut microbiota and subsequent risk of overweight in children. CMAJ. 2018;190(45):E1341.

16. Zang L, Ma Y, Huang W, Ling Y, Sun L, Wang X, et al. Dietary Lactobacillus plantarum ST-III alleviates the toxic effects of triclosan on zebrafish (Danio rerio) via gut microbiota modulation. Fish Shellfish Immunol. 2019;84:1157-69.

Publisher's note Springer Nature remains neutral with regard to jurisdictional claims in published maps and institutional affiliations. 